This is the pre-print (ie. pre-refereeing ) version of the following manuscript: Kopec JA, Sayre EC, Davis AM, Badley EM, Abrahamowicz M, Pouchot J, Sherlock L, Esdaile JM. Development of a paperand-pencil semi-adaptive questionnaire for 5 domains of health-related quality of life (PAT-5D-QOL). Qual Life Res. 2013 Dec;22(10):2829-42, which has been published in the final form at:

http://www.ncbi.nlm.nih.gov/pubmed/23653158

\title{
Development of a paper-and-pencil semi-adaptive questionnaire for 5 domains of health-related quality of life (PAT-5D-QOL)
}

\author{
${ }^{1,2}$ Jacek A. Kopec, ${ }^{2}$ Eric C. Sayre, ${ }^{3}$ Aileen M. Davis, ${ }^{4}$ Elizabeth M. Badley, ${ }^{5}$ Michal \\ Abrahamowicz M, ${ }^{6}$ Jacques Pouchot, ${ }^{7}$ Lesley Sherlock L, ${ }^{2,8}$ John M. Esdaile
}

${ }^{1}$ School of Population and Public Health, University of British Columbia, Vancouver, BC, Canada

${ }^{2}$ Arthritis Research Centre of Canada, Richmond, BC, Canada

${ }^{3}$ Health Care and Outcomes Research, Toronto Western Research Institute, University Health

Network and Institute of Health Policy, Management and Evaluation and Departments of

Rehabilitation Science and Physical Therapy, University of Toronto, Toronto, ON, Canada

${ }^{4}$ Dalla Lana School of Public Health, University of Toronto, Toronto, ON, Canada

${ }^{5}$ Department of Epidemiology, Biostatistics and Occupational Health, McGill University, Montreal, QB, Canada

${ }^{6}$ Hôpital européen Georges Pompidou, Université Paris Descartes, Assistance Publique Hôpitaux de Paris, Paris, France.

${ }^{7}$ City of Richmond, BC, Canada

${ }^{8}$ Division of Rheumatology, University of British Columbia, Vancouver, BC, Canada 


\section{Abstract}

Objectives: To develop a paper-and-pencil semi-adaptive test for 5 domains of health-related quality of life (PAT-5D-QOL) based on item response theory (IRT).

Methods: The questionnaire uses items from previously developed item banks for 5 domains: 1) walking, 2) handling objects, 3) daily activities, 4) pain or discomfort and 5) feelings. For each domain, respondents are initially classified into 4 functional levels. Depending on the level, they are instructed to respond to a different set of 5 additional questions. IRT scores for each domain and overall health utility scores are obtained using a simple spreadsheet. The questions were selected using psychometric and conceptual criteria. The format of the questionnaire was developed through focus groups and cognitive interviews. Feasibility was tested in two population surveys. A simulation study was conducted to compare PAT-5D-QOL with a computerized adaptive test (CAT-5D-QOL) and a fixed questionnaire, developed from the same item banks, in terms of accuracy, bias, precision, and ceiling and floor effects.

Results: Close to $90 \%$ of the participants in feasibility studies followed the skip instructions properly. In a simulation study, scores on PAT-5D-QOL for all domains tended to be more accurate, more precise, less biased and less affected by a ceiling effect than scores on a fixed IRT-based questionnaire of the same length. PAT-5D-QOL was slightly inferior to a fully adaptive instrument.

Conclusions: PAT-5D-QOL is a novel, semi-adaptive, IRT-based measure of health-related quality of life with a broad range of potential applications.

Keywords: Computerized adaptive testing, Questionnaires, Health related quality of life, Measurement, Item response theory 


\section{Introduction}

One of the most important applications of item response theory (IRT) in health and quality of life assessment is computerized adaptive testing (CAT) [1,2]. In CAT, different respondents answer different questions, selected dynamically by a computer. Question selection is based on the subject's responses to previous questions. The algorithm for question selection uses the properties of each question stored in a database (item bank). Usually, an IRT score for the measured attribute is estimated according to a pre-specified IRT model after each response, and the most informative question at the estimated attribute level is selected next [3-4]. A number of item banks for different health-related attributes have been developed and several CAT systems are available [5-11].

CAT scores are more precise and less biased than scores from a standard "fixed" questionnaire of the same length $[3-6,10]$. However, a major limitation of CAT is the need for computer access and special software for questionnaire administration and scoring. In many studies, a paper-andpencil questionnaire, either mailed or handed out to the participants, is the only feasible method of data collection. Even when the questionnaire is administered on a computer (usually online), users may not have access to CAT software or may prefer a conventional platform that allows inhouse administration and scoring. Therefore, an important question is whether CAT technology can be adapted to a paper-and-pencil questionnaire.

In theory, it is possible to develop a paper-based adaptive questionnaire of any length or complexity by providing appropriate skip instructions (branching). Indeed, the principles of adaptive assessment (tailored testing) were known before the wide availability of computers [12- 
14]. A simplified, conventionally scored version of an adaptive ability test with dichotomous items, known as a flexilevel test, was proposed by Lord in 1971 [15]. However, for an adaptive, paper-based health-related quality of life (HRQOL) instrument to be practical the skip instructions cannot be too complicated and the number of pages has to be reasonable. The complexity and length of an adaptive questionnaire increases dramatically as the number of steps that require skip logic becomes greater than two. A two-stage IRT-based test was discussed by Lord [16]. Cook et al [17] implemented this approach to measure shoulder function.

Our goal was to develop a two-step paper-based "semi-adaptive" test for 5 domains of HRQOL (PAT-5D-QOL). The underlying idea was as follows: In step 1, the respondents would answer a single, multi-option routing question to classify themselves into one of several levels of function for a given domain. In step 2, they would respond to a set of multi-option items for that domain that would depend on the answer in step 1, i.e., items would be different for each level of the domain as determined in step 1 .

The development of PAT-5D-QOL paralleled the development of 5 item banks for a computerized adaptive test (CAT-5D-QOL). In the current study we: 1) describe the development of a suitable format for PAT-5D-QOL; 2) select the items from the CAT-5D-QOL item banks; and 3) compare PAT-5D-QOL with a fixed IRT-based questionnaire and a full CAT in a simulation study. 


\section{Methods}

\section{Item banks}

In a previously published study [18], we developed IRT-based item banks for 5 domains of HRQOL. The domains are Walking (WALK), Handling Objects (HAND), Daily Activities (DAILY), Pain or Discomfort (PAIN), and Feelings (FEEL). Details of item development, reduction and calibration have been described by Kopec et al [18] and are briefly summarized here. Key steps in the development of PAT-5D-QOL and CAT-5D-QOL are presented in Figure 1.

A database of 1400 items obtained from widely used questionnaires was developed. The items were classified into 19 domains using concepts from the International Classification of Function, Disability and Health (ICF) [19]. Of those, 624 items from 5 domains considered critical for the assessment of HRQOL were selected for further evaluation. Through an iterative evaluation process, redundant or poorly worded items were eliminated and new items were drafted to fill gaps in concept coverage and difficulty levels. The items were reworded using a standardized recall period and response format. A resultant pool of 230 items underwent cognitive testing through focus groups and individual interviews. As a result, the investigators identified 221 items that were included in a psychometric item calibration study.

Participants in the item calibration study were patients drawn from two clinics at the Vancouver Hospital and Sciences Centre (VHSC) and a stratified random community sample in British Columbia (BC), Canada [18]. Data were obtained from 1,666 individuals (331 rheumatology patients, 340 orthopedic patients, and 995 subjects from an age-stratified community sample). 
Extensive analyses included dimensionality assessment through exploratory factor analysis, differential item functioning (DIF) analysis using ordinal logistic regression, and IRT analyses based on the generalized partial credit model (GPCM) [20]. Item parameters were estimated from the GPCM using PARSCALE [21]. Item fit was assessed by comparing empirical and model-based option characteristic curves and computing a chi-square test of fit for each item. Items that showed significant and substantial DIF by age or sex and items that did not fit the IRT model were deleted. Additional items were dropped due to content overlap or redundancy. In the final version, 159 items were retained (WALK - 30, HAND - 44, DAILY - 23, PAIN - 28, and FEEL - 34). These item banks were used to develop CAT-5D-QOL, as described in a prior publication [22], and the paper-based test (PAT-5D-QOL) discussed in the current article.

\section{Format development: Qualitative studies}

The PAT-5D-QOL required a novel questionnaire design to ensure that the instructions were easy to follow and the questionnaire was not cumbersome. Initially, five designs were developed. They presented different ways of linking the domain level (step 1) with step-2 questions, hence different visual presentations and instructions were required. Legal-size and landscape layouts, as well as the standard letter-size portrait layout, were tested. Four focus groups with a total of 23 subjects were conducted, with participants recruited through posters and newspaper advertisements. The two preferred layouts did not require participants to skip pages to connect second-tier with first-tier responses. These layouts were tested through individual cognitive interviews with 10 subjects. Interviews resulted in the selection and refinement of a format presenting both tiers on one sheet, so that each domain was contained on a single page. In the first-tier questions in each of the five domains, four response choices were used. 
The maximum number of tier-two questions that would fit on one page, with acceptable font and spacing, was 5 questions per level (20 questions total). For greater clarity, each set of 5 tier-two questions was enclosed in a box. There were 4 boxes per page (domain), labeled A, B, C and D, corresponding to the four response options (levels of health) in the tier-one questions. Seven individuals aged 69 to 83 tested the final format of the questionnaire. All but one were able to complete the questionnaire correctly without any help.

\section{Feasibility testing: Version 0}

In the next step, we evaluated the feasibility and ease of implementation of the PAT-5D-QOL in a general population survey. Subjects for the study were selected randomly from the population of telephone subscribers in British Columbia, Canada. We asked the oldest person in the household to fill out a questionnaire that included the PAT-5D-QOL and, for comparison, the SF-36 Health Survey [23]. Because the feasibility survey was performed before the results of the item calibration study were available, the items in each domain were selected intuitively, rather than based on their psychometric properties (Version 0). Nonetheless, the items were similar to other items within each domain in terms of general format, length, response options, and concepts measured.

\section{Further feasibility testing: Version 1}

Our next objective was to assess the feasibility of a new version of the instrument among persons with a chronic health problem. In particular, we were interested in the frequency of errors in completing the questionnaire. A new set of items for PAT-5D-QOL was developed using preliminary data from the item calibration study (Version 1). The main criteria for item selection 
were item information and location (difficulty), coverage of different facets of each domain, and response scale types. This version was used in a large mail population survey of persons diagnosed with osteoarthritis (OA) in British Columbia, Canada. Details of the survey have been published [24]. PAT-5D-QOL was part of a larger questionnaire that included questions about pain, chronic conditions, diagnosis of OA, medical and surgical treatment, as well as functional health.

\section{Item selection for Version 2}

PAT-5D-QOL Version 2 (current version) was developed from items included in the final CAT5D-QOL item banks. The format of the questionnaire is not substantially different from Version 1. The main difference is a slight modification of the skip instructions. The criteria for item selection were item information, item location (difficulty), as well as content coverage and type of response scale, as described below.

In each domain-specific item bank, one question has a special status. This question, referred to as the routing question, is used to classify the respondents into 4 levels of functional health for a given domain. (It may be noted that the same questions are used as routing items in the fully adaptive CAT-5D-QOL). In step 2, each response option is followed by a set of 5 questions, as previously discussed. To identify the best set of questions for each functional level, independently for each domain, we defined 6 ordered IRT scores: minimum estimable score, scores for each response category of the routing item (4 scores) and the maximum estimable score. This produced the following four overlapping intervals used for item selection within the four levels: scores 1 to 3 (level A), scores 2 to 4 (level B), scores 3 to 5 (level C), and scores 4 to 
6 (level D). On the Handling Objects domain, there are only 3 intervals because the initial item is collapsed for the purpose of IRT scoring (lowest two categories are treated the same).

The maximum/minimum estimable IRT score for a domain was defined as the score obtained when a respondent answers every question in that domain in the full item bank, and selects the highest/lowest category for every question except one, on which they select the second highest/lowest category. IRT scores and standard errors were calculated for each level of the four-level routing item. For the non-extreme levels (two middle levels), these are obtained as maximum likelihood estimates. For the extreme levels, the score and standard error were defined to be the mean and standard error in the segment of the study sample who selected the extreme category, based on the full item bank.

Items for each interval were selected using both psychometric and conceptual criteria. Feasibility of different response types within each level was also taken into account. The psychometric criteria included item information and location (difficulty). All items in each domain were rankordered according to area under the information curve within the interval. Items were considered for selection in the order of information. For the two extreme levels, floor and ceiling effects were addressed by including the lowest/highest item by location in each domain. If the lowest/highest item was collapsed, the second lowest/highest item was evaluated for inclusion.

Additional considerations involved the content of the items. Within each domain, we covered different "facets" of that domain. For example, in WALK, we included items about walking various distances, running, climbing stairs, and standing. At the same time, we did not allow two 
or more items that were very similar in content and level of activity, and differed only by the type of response scale (e.g., difficulty vs. limitation).

Another consideration was the selection of content-appropriate items for the extreme levels of the domains, such as "unable to walk". Our data showed that many participants who initially selected such extreme responses were actually able to perform "low-difficulty” activities. This was reflected in the selection of the items. For example, the lowest level of walking includes items that do not involve walking, such as sitting up in bed or standing with/without support, as well as items asking about walking and climbing a few steps. Similarly, in PAIN, participants who initially selected "no pain or discomfort” may have been limited by pain/discomfort in highdifficulty activities, and such questions were included according to item information ranking.

Finally, in the lowest level of DAILY and the highest level on the PAIN domain (least pain), items with two different response types were allowed. For example, in the lowest level of DAILY, we included questions about difficulty and questions about need for help with specific activities. However, allowing for more than two response types was not feasible given the format of the questionnaire. The final version of the PAT-5D-QOL questionnaire is displayed in the Appendix.

\section{Comparisons of PAT-5D-QOL with CAT-5D-QOL and a fixed questionnaire}

We performed a simulation study to assess the properties of the final PAT-5D-QOL (Version 2) against CAT-5D-QOL and a fixed IRT-based questionnaire (FQ-5D-QOL). The "best" fixed questionnaire for comparison was selected using psychometric criteria. Five intervals of IRT 
scores were defined for each domain by partitioning the domain's range into five equal parts. The CAT-5D-QOL initial item was included, to provide good coverage across the entire range. The remaining items were selected according to maximum information in each of the four intervals.

The simulation study used item calibration data from the CAT-5D-QOL item banks. We simulated 1000 subjects (response patterns). "True” IRT scores (latent trait) for each domain were assigned evenly from minimum to maximum estimable IRT score. Random responses to each item were assigned based on the known response probabilities (option characteristic curves) for each level of true score from the item calibration data. CAT-5D-QOL was simulated using a standard algorithm as described by Kopec et al [22], in which the item with the highest information for the estimated score is selected, starting with the routing item. Six items were administered in each domain. PAT-5D-QOL was simulated by first simulating the response to the routing item, followed by responses to the 5 items for the domain level selected in step 1 . For the FQ-5D-QOL, responses to the 6 a priori selected items were simulated.

The simulation was performed for each of the 5 domains. In addition, we simulated 3 different score distributions in the population using kernel weighting. The score distributions simulated a "standard" population (normal distribution kernel), a healthy population with a distribution skewed to left (a beta distribution kernel) and an unhealthy population skewed to the right (also a beta distribution kernel). The measures were compared according to: 1) score accuracy as measured by mean absolute difference between the estimated score and "true" score; 2) bias, i.e., mean difference between the estimated and true score; 3) precision (width of IRT-based 95\% 
confidence interval around the estimated score); and, 4) ceiling effect (proportion of subjects with maximum score) and floor effect (proportion of subjects with minimum score).

\section{Results}

\section{Feasibility study with PAT-5D-QOL Version 0}

Data were obtained from 199 participants (40\% response rate). Nine subjects (5\%) did not follow the skip instructions correctly (e.g., completed more than one "box" per page, answered only one question per box). Very few respondents $(\mathrm{n}=3$ ) expressed confusion about the two-step format of the questionnaire. Compared with the SF-36, slightly more people found the PAT-5DQOL instructions very clear (PAT-5D-QOL, 89\%; SF-36, 81\%). The proportion of subjects needing help in filling out the questionnaire was similar for both instruments (PAT-5D-QOL, 8\%; SF-36, 7\%). The average self-reported completion time for the semi-adaptive assessment was 10 minutes, averaging 1.67 minutes per domain (14-15 seconds per item).

\section{Feasibility study with PAT-5D-QOL Version 1}

Data for the second feasibility study were obtained from 1,349 individuals with physiciandiagnosed osteoarthritis. The mean age was 67 years, $60 \%$ were female, $29 \%$ had above high school education, and 39\% were in fair or poor health. Overall, 84\% followed skip instructions correctly for all domains, 7\% responded to multiple levels on one domain, with the remaining 9\% responding to multiple levels on more than one domain. The results by domain (Table 1) suggest that the proportion of correct responses was the lowest for the domain presented first and highest for the domain presented last. These results provided reassurance that the questionnaire is feasible to use in large general population surveys. 


\section{Simulation study with PAT-5D-QOL Version 2}

Scores on all 3 measures in all domains were highly correlated with true scores. All correlation coefficients were $>0.97$. For all domains, the highest correlation coefficients were observed for CAT-5D-QOL and the lowest for FQ-5D-QOL. Agreement with true scores for all measures was the strongest in the middle of the score range and slightly weaker for very low and very high scores (data not shown).

Comparisons between the CAT-5D-QOL, PAT-5D-QOL and FQ-5D-QOL are presented in Table 2. The accuracy, as measured by the mean absolute error was smaller for PAT-5D-QOL than FQ-5D-QOL in 4 out of 5 domains in the standard population, all 5 domains in the healthy population, and 4 out of 5 domains in the unhealthy population. The mean absolute error was almost always the smallest for CAT-5D-QOL; however, the amount of error for PAT-5D-QOL was in most cases closer to CAT-5D-QOL than FQ-5D-QOL. In fact, the error for PAT-5D-QOL and CAT-5D-QOL was very similar or equal in 2 domains in the standard population, 3 domains in the healthy population, and 3 domains in the unhealthy population.

Bias was generally small for all measures except FQ-5D-QOL in the healthy population, and was smaller for PAT-5D-QOL than FQ-5D-QOL for 3 domains in the standard population and all domains in the healthy population (Table 2). In the unhealthy population, PAT -5D-QOL had less bias than FQ-5D-QOL in 2 domains and equal bias in 3 domains. CAT-5D-QOL had the least bias in most scenarios, but the difference compared with PAT-5D-QOL appeared small $(<0.2)$ in more than half of the scenarios. Scores were more likely to be underestimated than 
overestimated by PAT-5D-QOL in all domains in the healthy population, 4 domains in the unhealthy population and 2 in the standard population. FQ-5D-QOL showed a stronger tendency to underestimate the scores than PAT-5D-QOL, especially in the healthy population, in which the average amount of bias ranged from -1.8 for PAIN to -6.8 for FEEL, compared to - 0.4 to 1.4, respectively, for PAT-5D-QOL.

The 95\% IRT-based confidence intervals (CIs) were narrower for PAT-5D-QOL compared to FQ-5D-QOL for all domains in the standard population, 3 domains in the healthy population, and all domains in the unhealthy population (Table 2). In the healthy population, PAT-5D-QOL had slightly wider CIs than FQ-5D-QOL for HAND and FEEL. Confidence intervals were the smallest for CAT-5D-QOL in all domains in the standard population, 4 domains in the healthy population and 4 domains in the unhealthy population.

The ceiling effect was considerably smaller for PAT-5D-QOL compared to FQ-5D-QOL for all domains in both standard and healthy populations (Table 2). In the healthy population, where this problem was especially pronounced, ceiling effects for PAT-5D-QOL and FQ-5D-QOL were 27\% vs. $41 \%$ for WALK, 30\% vs. 37\% for HAND, 20\% vs. 35\% for DAILY, 25\% vs. 33\% for PAIN and 30\% vs. 60\% for FEEL, respectively. Ceiling effects were the smallest for CAT-5DQOL. In terms of the size of the ceiling effects, PAT-5D-QOL was more similar to CAT-5DQOL than to FQ-5D-QOL in 4 domains in the standard population and 3 domains in the healthy population. There were no substantial ceiling effects in the unhealthy population for any of the instruments. Floor effects were not a problem for any of the instruments (0.0-0.1\% for CAT-5DQOL, 0.0-0.9\% for PAT-5D-QOL and 0.0-2.2\% for FQ-5D-QOL). 


\section{Discussion}

We have developed a paper-and-pencil, IRT-based questionnaire for 5 domains of health-related quality of life (PAT-5D-QOL). The domains cover key concepts commonly assessed in outcomes research, such as physical function (upper and lower body), daily activities, symptoms (pain and discomfort), and emotional health. The questions for PAT-5D-QOL were selected from previously validated and calibrated item banks. The final items had good fit to the generalized partial credit model and were free of significant DIF. In the selection of items for the paperbased questionnaire, the main criteria were item information, item difficulty, ceiling and floor effects, as well as domain coverage, redundancy and response types.

PAT-5D-QOL is not fully adaptive. In a CAT-based questionnaire, every item (except the first one) is selected dynamically, based on answers to all previous questions. In our semi-adaptive instrument, for each of the 5 domains respondents are first asked to choose one of 4 levels of function and, depending on the level selected, are instructed to answer 5 additional questions. Through focus groups and cognitive interviews we have developed a novel questionnaire design to ensure that the instructions are clear and the questionnaire is easy to fill out. Each domain is presented on a single page. The average completion time is about 10 minutes.

Each domain of the PAT-5D-QOL is scored separately and independently using IRT methodology, as described for CAT-5D-QOL [18,22]. The scores for each possible response pattern have been computed and are easily obtained using an Excel spreadsheet provided with the questionnaire. Since PAT-5D-QOL is a selection of items from CAT-5D-QOL, the scores are equivalent. The scoring methods developed for the fully adaptive measure, such as population- 
based scoring of the domains are, therefore, applicable. It should be noted that a method for combining domain-specific IRT scores for the CAT-5D-QOL into an overall utility score has been developed [25] and the same formula can be applied to scores from PAT-5D-QOL.

As demonstrated in our simulation study, the main advantages of a semi-adaptive measure, compared to a "fixed" IRT-based questionnaire of the same length, is greater accuracy of the scores. Specifically, both the mean absolute error and mean error tend to be smaller, confidence intervals tend to be narrower, and ceiling effects are significantly reduced. On a standard normbased scale with a mean of 50 and SD of 10, the average mean absolute error across 5 domains and 3 population types was 2.3 for CAT-5D-QOL, 2.5 for PAT-5D-QOL, and 3.2 for FQ-5DQOL. Thus, using the fixed questionnaire would increase the mean absolute error by $28 \%$ compared with the semi-adaptive measure and by 39\% compared with the full CAT. The average width of the 95\% CI for the three measures was 9.9, 10.6, and 11.4, respectively (Table 2), while the average ceiling effect was $7.8 \%, 11.1 \%$ and $17.6 \%$, respectively. The latter advantage is especially important when studying relatively healthy subjects. These advantages are likely to be even greater when compared with standard instruments developed using conventional (classic) measurement methods. However, more experience with the instrument is needed to determine how often, and under what circumstances, the improved measurement properties would translate into better clinical decisions or improved interpretation of research data. The scores on PAT-5DQOL are generally less accurate than CAT-5D-QOL scores (for the same number of items) although the difference often seems relatively small. 
There are some limitations to our study. First, evidence of feasibility has been obtained for Version 1 only. However, given the considerable overlap in content between Versions 1 and 2, careful selection of the items from validated item banks, and improved instructions, it seems unlikely Version 2 is inferior to Version 1. Second, psychometric properties of the instrument have only been assessed in a simulation study, rather than an empirical head-to-head comparison with other measures.

The idea of an adaptive questionnaire that could be administered on paper is not new. More than 40 years ago, Lord proposed a flexilevel test that could be scored using conventional methods [15]. The properties of flexilevel tests were studied by Beth and Weiss [26], Ayala [27], and Lilley and Piper [28] in the context of educational measurement. Lord also suggested a two-stage IRT-based test [16]. Cook et al [17] developed a two-stage shoulder function questionnaire called FLEX-SF, in which the respondents are instructed to answer one of three testlets (easy, medium difficulty and hard) based on their answer to a routing question.

Our study shows that a generic semi-adaptive health questionnaire is feasible and that its measurement properties, as one would expect, are intermediate between a fixed questionnaire and a full CAT of the same length. When a paper-based questionnaire is the only option, PAT5D-QOL could be considered an alternative to a conventional, generic (multi-domain) instrument. Potential advantages of PAT-5D-QOL are better discrimination and responsiveness for relatively healthy as well as very unhealthy individuals (smaller ceiling or floor effects), although these advantages need to be demonstrated empirically in head-to-head comparisons. Moreover, selected domains of PAT-5D-QOL can be used to measure specific concepts of 
HRQOL. In this regard, the instrument should be considered an alternative to various domainspecific instruments, such as those measuring lower and upper extremity function, pain, or emotional function. How the PAT-5D-QOL domains would perform against established measures of similar concepts remains to be elucidated. Based on our simulation results, the semiadaptive questionnaire is likely to provide more accurate scores, with less ceiling effect. However, these comparisons pertain only to the items included in the 5 item banks of the CAT5D-QOL. Alternative measurement systems and corresponding item banks, such as those from the Patient-Reported Outcomes Measurement Information System (PROMIS) [5,29] have been developed, including IRT-based fixed questionnaires. It will be important, in our view, to carry out direct comparisons of the PAT-5D-QOL domains against short IRT-based, fixed instruments.

The PAT-5D-QOL domains can also be considered an alternative to disease-specific measures, especially in musculoskeletal conditions and other conditions in which the domains are relevant. Head-to-head comparisons in patients with a variety of conditions will be needed to determine if the semi-adaptive questionnaire is more discriminating and responsive to change than standard disease-specific instruments.

If the questionnaire is to be administered on a computer, PAT-5D-QOL can be presented using simple skip logic available in all commercial online survey systems (e.g., SurveyMonkey). Since computer administration eliminates any potential problems in following skip instructions, and IRT-based scoring is done with an Excel spreadsheet, a computer-administered PAT-5D-QOL is as convenient to use as a fixed questionnaire, while providing more accurate scores. PAT-5DQOL is also a reasonable alternative to a full CAT, because it does not require potentially 
expensive CAT software for administration and scoring. Only a handful of validated multidomain CAT systems for measuring HRQOL are widely available [6,22,29-31] and many researchers unfamiliar with this technology may prefer a more standard platform for questionnaire administration. Of course, CAT offers more flexibility in the number of questions administered and the possibility to apply a variety of stopping rules, for example, based on score accuracy.

The questionnaire is feasible, as demonstrated in two large, mail population surveys. In one of the surveys, PAT-5D-QOL was as easy to fill out as the SF-36. Nonetheless, about 5-15\% of the subjects in our feasibility studies using Versions 0 and 1 of the questionnaire did not follow the skip instructions properly and answered more questions than needed. This proportion is likely smaller for Version 2 due to improved instructions, although this has yet to be demonstrated. It should be emphasized that the scoring of subjects who answer additional questions or do not follow skip instructions is straightforward and does not invalidate the results (these are not missing data). Scoring guidelines for such respondents have been developed. Indeed, in contrast to standard measures, a response to a single item is sufficient to compute a valid score using the scoring spreadsheet. One potential limitation of PAT-5D-QOL in some studies is that each domain needs to be presented on a separate page, which may slightly increase the total number of pages. However, presenting each domain-specific measure on a separate page is a common practice and is probably desirable in most situations.

Some potential users might wonder whether a longer, fixed questionnaire would be as effective as PAT-5D-QOL, with little loss in efficiency and a simplified format. The total number of 
unique questions per domain (including the routing item) in PAT-5D-QOL is 18 in WALK, 16 in HAND, 16 in DAILY, 15 in PAIN and 17 in FEEL. In terms of psychometric properties, a questionnaire that includes all these items (82 items total) would likely perform at least as well as PAT-5D-QOL. However, gain in performance when the number of items increases beyond 5 becomes relatively small, as shown in Figure 2 for CAT-5D-QOL, even if the most informative items are used. Asking additional items that are inappropriate for the level of health would likely have a marginal effect on measurement characteristics. At the same time, some questions would appear repetitive or redundant to respondents, and many would appear irrelevant. In terms of efficiency, a questionnaire with 82 items would take about 25-30 minutes to administer, compared with 10 minutes for the semi-adaptive instrument. Finally, it would be impossible to score such a questionnaire using a simple spreadsheet because the number of possible response patterns increases exponentially with the number of items.

In conclusion, the PAT-5D-QOL is a novel, semi-adaptive HRQOL instrument with a broad range of potential applications. Our simulation study demonstrated its advantages compared with a "fixed" IRT-based instrument. Further empirical research is needed to compare the reliability, validity and responsiveness of this measure with other IRT-based instruments, including fixed domain-specific measures from PROMIS, as well as standard generic and disease-specific measures. 


\section{Acknowledgements}

Many people have helped with the development and testing of PAT-5D-QOL. The authors would like to thank Aslam Anis, Sherry Bar, Andrew Chalmers, Jolanda Cibere, Donald Garbuz,

Nelson Greidanus, Fayez Al-Harthy, James Latteier, Linda Li, Viviane Dias Lima, and Carlo Marra. 


\section{References}

1. Revicki DA, Cella DF. Health status assessment for the twenty-first century: Item response theory, item banking and computer adaptive testing. Qual Life Res 1997; 6(6):595-600.

2. Hays RD, Morales LS, Reise SP: Item response theory and health outcomes measurement in the 21st century. Med Care 2000, Suppl 9:II28-42.

3. Wainer H. Computerized Adaptive Testing: A primer. Hillsdale, NJ: Lawrence Erlbaum Associates, Publishers, 1990.

4. Embretson S, Reise SP. Item Response Theory for Psychologists. Mahwah, NJ: Lawrence Erlbaum Associates, 2000.

5. Fries JF, Bruce B, Cella D: The promise of PROMIS: using item response theory to improve assessment of patient-reported outcomes. Clin Exp Rheumatol 2005, 23:S53-57.

6. Ware JEJr, Kosinski M, Bjorner JB, Bayliss MS, Batenhorst A, Dahlöf CGH, Tepper S, Dowson A. Applications of computerized adaptive testing (CAT) to the assessment of headache impact. Qual Life Res 2003;12(8):935-952.

7. Bode RK, Lai JS, Dineen K, Heinemann AW, Shevrin D, Von Roenn J, Cella D. Expansion of a physical function item bank and development of an abbreviated form for clinical research. J Appl Meas 2006;7:1-15.

8. Hart DL, Mioduski JE, Stratford PW. Simulated computerized adaptive tests for measuring functional status were efficient with good discriminant validity in patients with hip, knee, or foot/ankle impairments. J Clin Epidemiol 2005;58:629-38.

9. Hart DL, Mioduski JE, Mark W, Werneke MW, Stratford PW. Simulated computerized adaptive test for patients with lumbar spine impairments was efficient and produced valid measures of function. J Clin Epidemiol 2006;59:947-956.

10. Lai JS, Dineen K, Reeve BB, Von Roenn J, Shervin D, McGuire M, Bode RK, Paice J, Cella D. An item response theory-based pain item bank can enhance measurement precision. J Pain Symptom Manage 2005;30:278-88.

11. Turner-Bowker DM, DeRosa MA, Saris-Baglama RN, Bjorner JB. Development of a computerized adaptive test to assess health-related quality of life in adults with asthma. $J$ Asthma. 2012 Mar;49(2):190-200. Epub 2011 Nov 24.

12. Lord FM. Tailored testing: An application of stochastic approximation. J Am Stat Assoc. Vol. 66, No. 336, Dec., 1971 
13. Urry VW. Tailored testing: A successful application of latent trait theory. J Educational Measurement 1977;14:181-186.

14. Lord FM. Applications of Item Response Theory to Practical Testing Problems. Hillsdale, NJ: Lawrence Erlbaum Associates, Publishers, 1980.

15. Lord FM. The self-scoring flexilevel test. J Educational Measurment 1971, 8(3); 147-151

16. Lord FM. A theoretical study of two-stage testing. Psychometrika. 1971; 36 (3), 227-241

17. Cook KF, Roddey TS, Gartsman GM, Olson SL. Development and psychometric evaluation of the Flexilevel Scale of Shoulder Function. Med Care. 2003 Jul;41(7):82335.

18. Kopec JA, Sayre EC, Davis AM, Badley EM, Abrahamowicz M, Sherlock L, Williams JI, Anis AH, Esdaile JM. Assessment of health-related quality of life in arthritis: Conceptualization and development of five item banks using item response theory. Health Qual Life Outcomes 2006;4:33.

19. Ustun TB, Chatterji S, Bickenbach J, Kostanjsek N, Schneider M: The International Classification of Functioning, Disability and Health: a new tool for understanding disability and health. Disabil Rehabil 2003, 25:565-571.

20. Muraki E. A Generalized Partial Credit Model. In: van der Linden WJ, Hambleton RK. Handbook of Modern Item Response Theory. New York: Springer, 1996: pp. 153-164.

21. Muraki E, Bock RD. Parscale: IRT based test scoring and item analysis for graded openended exercises and performance tasks. Chicago: Scientific Software Int, 1993.

22. Kopec JA, Badii M, McKenna M, Lima VD, Sayre EC, Dvorak M. Computerized adaptive testing in back pain: Validation of CAT-5D-QOL. Spine. 2008;33:1384-90.

23. Ware JE, Sherbourne CD. The MOS 36-item Short Form Health Survey (SF-36). Med Care 1992;30:473-483.

24. Sayre EC, Li LC, Kopec JA, Esdaile JM, Bar S, Cibere J. The effect of disease site (knee, hip, hand, foot, lower back or neck) on employment reduction due to osteoarthritis. PLoS One. 2010;5(5):e10470

25. Sayre EC, Kopec JA, Abrahamowicz M, Anis AH, Badley EM, Davis AM, Esdaile JM. Modeling multi-attribute health utility from five domain-specific IRT scores. Qual Life Res 2007;(Suppl. A-49) (Abstract 1598). Presented at the 14th Annual Scientific Meeting of the International Society for Quality of Life Research, Toronto, October 10-13, 2007.

26. Betz NE, Weiss DJ. Empirical and simulation studies of flexilevel ability testing. Research report 75-3, Psychometric Methods Program, Department of Psychology, 
University of Minnesota, Minneapolis, MN 55455, July 1975

27. De Ayala RJ, Dodd BG, Koch WR. A simulation and comparison of flexilevel and bayesian computerized adaptive testing. J Educational Measurement. 1990, 27 (3); 227239

28. Lilley M, Pyper A. The application of the flexilevel approach for the assessment of computer science undergraduates. In: J.A. Jacko (Ed.): Human-Computer Interaction, Part IV. Springer-Verlag Berlin Heidelberg 2009, pp. 140-148.

29. Carle AC, Cella D, Cai L, Choi SW, Crane PK, Curtis SM, Gruhl J, Lai JS, Mukherjee S, Reise SP, Teresi JA, Thissen D, Wu EJ, Hays RD. Advancing PROMIS's methodology: Results of the third PROMIS Psychometric Summit. Expert Review of Pharmacoeconomics and Outcomes Research, 2011, 11: 677-84.

30. Bjorner JB, Chang CH, Thissen D, Reeve BB. Developing tailored instruments: item banking and computerized adaptive assessment. Qual Life Res. 2007;16 Suppl 1:95-108.

31. Tulsky DS, Carlozzi NE, Cella D. Advances in outcomes measurement in rehabilitation medicine: current initiatives from the National Institutes of Health and the National Institute on Disability and Rehabilitation Research. Arch Phys Med Rehabil. 2011 Oct;92(10 Suppl):S1-6. 
Table 1: Percent of subjects who responded (correctly) to step-2 questions for the appropriate level of health and those who responded (unnecessarily) to questions for 2, 3 or all 4 levels in the survey of persons with osteoarthritis in British Columbia

\begin{tabular}{|l|c|c|c|c|}
\hline Domain & 1 level (correct) & 2 levels & 3 levels & 4 levels \\
\hline WALK & 90.5 & 3.3 & 1.8 & 4.5 \\
\hline HAND & 92.1 & 2.8 & 0.6 & 4.6 \\
\hline DAILY & 89.2 & 4.4 & 2.0 & 4.4 \\
\hline PAIN & 93.6 & 2.1 & 0.6 & 3.7 \\
\hline FEEL & 93.6 & 1.8 & 0.7 & 3.8 \\
\hline
\end{tabular}

The domains were presented in the following order: DAILY, WALK, HAND, PAIN, FEEL 
Table 2: Comparisons of scores on PAT-5D-QOL (PAT, Version 2) with CAT-5D-QOL (CAT) and FQ-5D-QOL (FQ) in 3 simulated populations of $n=1000$ (scores are norm-based with mean $=50$ and $\mathrm{SD}=10$ )

a. Accuracy (mean absolute error)

\begin{tabular}{|l|c|c|c|c|c|c|c|c|c|}
\hline & \multicolumn{3}{|c|}{ Standard population } & \multicolumn{3}{c|}{ Healthy population } & \multicolumn{3}{c|}{ Unhealthy population } \\
\hline & CAT & PAT & FQ & CAT & PAT & FQ & CAT & PAT & FQ \\
\hline WALK & 1.7 & 2.1 & 2.4 & 2.3 & 2.8 & 4.0 & 1.4 & 1.4 & 1.8 \\
\hline HAND & 2.5 & 2.7 & 3.1 & 3.0 & 3.0 & 3.7 & 1.6 & 1.7 & 2.0 \\
\hline DAILY & 2.2 & 2.3 & 3.1 & 2.3 & 2.4 & 3.6 & 1.7 & 1.9 & 2.4 \\
\hline PAIN & 2.2 & 2.3 & 2.7 & 2.6 & 2.7 & 3.1 & 1.9 & 1.8 & 2.3 \\
\hline FEEL & 2.6 & 2.8 & 2.8 & 3.9 & 4.1 & 7.2 & 2.7 & 2.9 & 3.3 \\
\hline
\end{tabular}

b. Bias (mean error)

\begin{tabular}{|l|c|c|c|c|c|c|c|c|c|}
\hline & \multicolumn{3}{|c|}{ Standard population } & \multicolumn{3}{c|}{ Healthy population } & \multicolumn{3}{c|}{ Unhealthy population } \\
\hline & CAT & PAT & FQ & CAT & PAT & FQ & CAT & PAT & FQ \\
\hline WALK & -0.1 & -0.2 & -0.7 & -0.8 & -1.4 & -3.1 & -0.1 & -0.1 & -0.1 \\
\hline HAND & 0.6 & 0.1 & 0.2 & 0.1 & -1.2 & -2.1 & 0.3 & 0.3 & 0.4 \\
\hline DAILY & -0.4 & -0.5 & -1.7 & -0.6 & -0.8 & -2.7 & -0.1 & -0.1 & -0.1 \\
\hline PAIN & 0.2 & 0.2 & -0.2 & -0.2 & -0.4 & -1.4 & -0.0 & -0.1 & -0.1 \\
\hline FEEL & -0.0 & 0.2 & -0.0 & -0.2 & -1.4 & -6.2 & -0.4 & -0.3 & -0.7 \\
\hline
\end{tabular}

c. Precision (width of the $95 \%$ confidence interval)

\begin{tabular}{|l|c|c|c|c|c|c|c|c|c|}
\hline & \multicolumn{3}{|c|}{ Standard population } & \multicolumn{3}{c|}{ Healthy population } & \multicolumn{3}{c|}{ Unhealthy population } \\
\hline & CAT & PAT & FQ & CAT & PAT & FQ & CAT & PAT & FQ \\
\hline WALK & 7.1 & 8.3 & 9.4 & 10.8 & 12.1 & 12.3 & 5.0 & 5.4 & 6.8 \\
\hline HAND & 10.1 & 11.6 & 12.6 & 14.9 & 16.8 & 16.2 & 5.7 & 6.1 & 7.8 \\
\hline DAILY & 9.9 & 10.2 & 11.5 & 11.4 & 11.6 & 12.7 & 6.6 & 7.1 & 8.2 \\
\hline PAIN & 8.8 & 9.2 & 10.6 & 12.4 & 12.6 & 12.9 & 6.9 & 6.9 & 8.7 \\
\hline FEEL & 9.6 & 10.6 & 11.3 & 18.7 & 19.0 & 17.6 & 10.3 & 11.0 & 13.0 \\
\hline
\end{tabular}

d. Ceiling (\% with maximum score)

\begin{tabular}{|l|c|c|c|c|c|c|c|c|c|}
\hline & \multicolumn{3}{|l|}{ Standard population } & \multicolumn{3}{l|}{ Healthy population } & \multicolumn{3}{c|}{ Unhealthy population } \\
\hline & CAT & PAT & FQ & CAT & PAT & FQ & CAT & PAT & FQ \\
\hline WALK & 2.9 & 5.1 & 9.7 & 18.9 & 27.2 & 41.2 & 0.0 & 0.0 & 0.0 \\
\hline HAND & 2.5 & 7.2 & 9.4 & 11.9 & 30.4 & 37.3 & 0.0 & 0.0 & 0.0 \\
\hline DAILY & 10.5 & 12.9 & 23.1 & 16.1 & 20.0 & 34.8 & 0.0 & 0.0 & 0.0 \\
\hline PAIN & 7.3 & 7.6 & 10.9 & 24.2 & 25.2 & 33.1 & 0.0 & 0.0 & 0.0 \\
\hline FEEL & 0.5 & 0.7 & 3.9 & 21.7 & 30.1 & 60.3 & 0.0 & 0.0 & 0.1 \\
\hline
\end{tabular}

e. Floor (\% with minimum score)

\begin{tabular}{|l|c|c|c|c|c|c|c|c|c|}
\hline & \multicolumn{3}{|c|}{ Standard population } & \multicolumn{3}{c|}{ Healthy population } & \multicolumn{3}{c|}{ Unhealthy population } \\
\hline & CAT & PAT & FQ & CAT & PAT & FQ & CAT & PAT & FQ \\
\hline WALK & 0.0 & 0.0 & 0.0 & 0.0 & 0.0 & 0.0 & 0.0 & 0.0 & 0.0 \\
\hline HAND & 0.0 & 0.0 & 0.0 & 0.0 & 0.0 & 0.0 & 0.0 & 0.0 & 0.0 \\
\hline DAILY & 0.0 & 0.0 & 0.0 & 0.0 & 0.0 & 0.0 & 0.0 & 0.0 & 0.0 \\
\hline PAIN & 0.0 & 0.0 & 0.0 & 0.0 & 0.0 & 0.0 & 0.0 & 0.2 & 0.0 \\
\hline FEEL & 0.0 & 0.0 & 0.0 & 0.0 & 0.0 & 0.0 & 0.1 & 0.9 & 2.2 \\
\hline
\end{tabular}


Figure 1: Stages in the development of PAT-5D-QOL and the parallel development of CAT-5DQOL.

PAT-5D-QOL

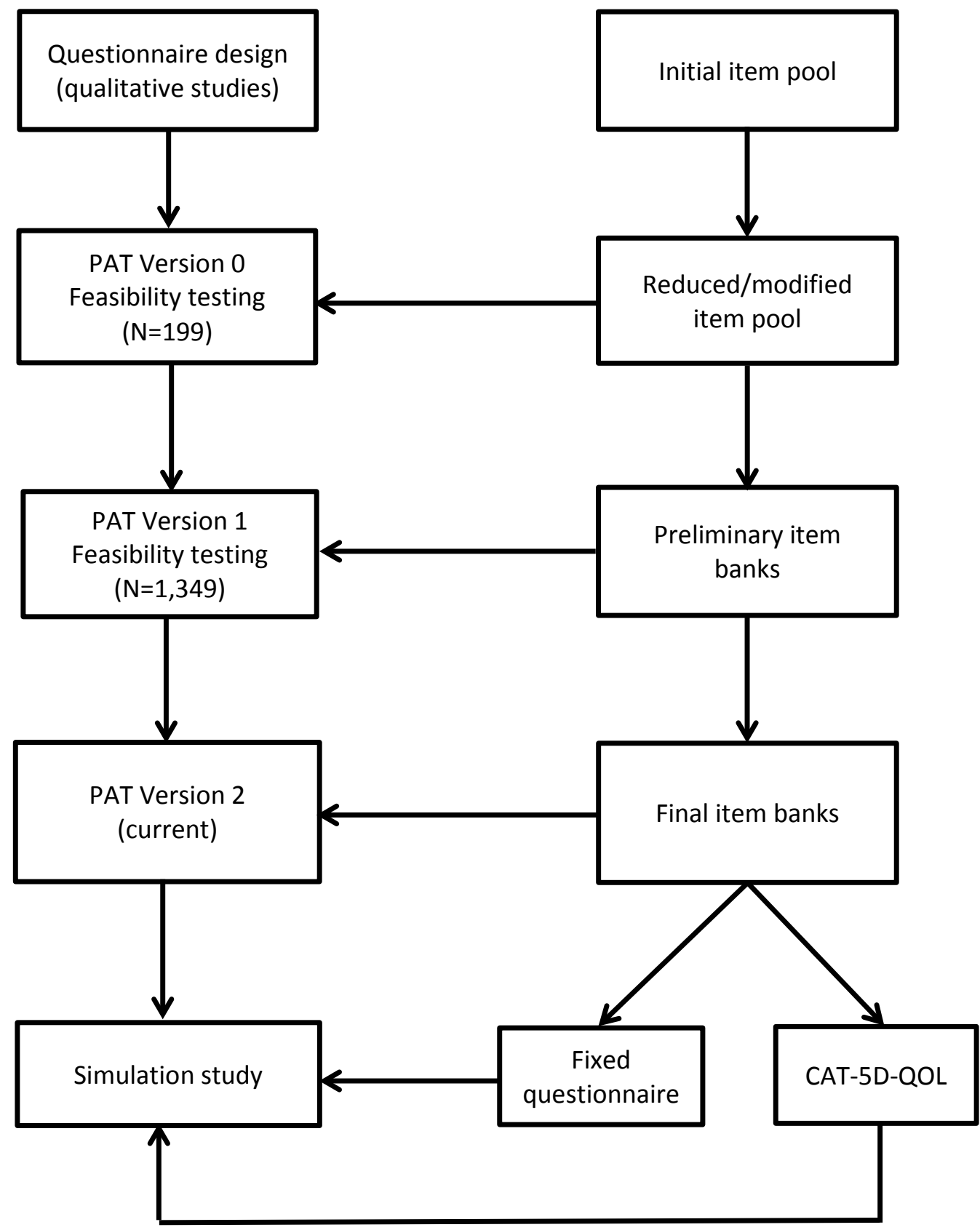


Figure 2: Mean absolute error for each domain of the CAT-5D-QOL as a function of the number of questions.

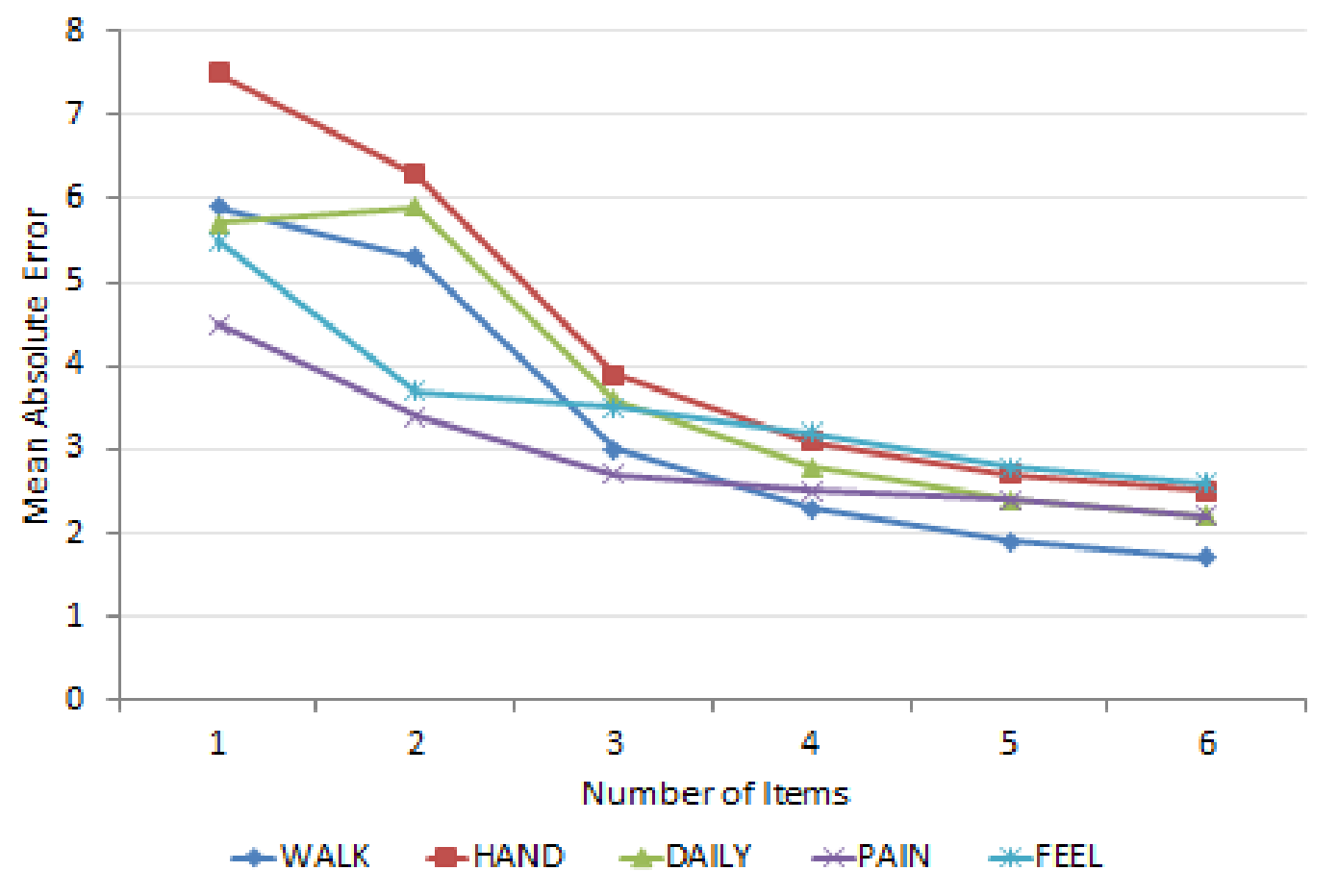


Appendix 1: The Paper-based Adaptive Test for 5 Domains of Health-related Quality of Life (PAT-5D-QOL)

\section{Attention: Check GO TO instructions for this question. Please read the statements below and check the one that describes you best in the past 4 weeks.}

I have no problems walking

I have a few problems walking

I have a lot of problems walking

I am unable to walk $\rightarrow$ Go to Box A on this page

$\rightarrow$ Go to Box B on this page

$\rightarrow$ Go to Box C on this page

$\rightarrow$ Go to Box D on this page

Please answer only questions in one of the boxes below, then move to next page A Answer questions in this box if you have no problems walking.

During the past 4 weeks, how difficult would it have been for you to...

1. Walk 10 miles (about 4 hours)

2. Run or jog 2 miles

3. Walk briskly

4. Be on your feet for 4 hours

5. Run or jog 20 miles

$\begin{array}{ccccc}\begin{array}{c}\text { Not difficult } \\ \text { at all }\end{array} & \begin{array}{c}\text { A little } \\ \text { difficult }\end{array} & \begin{array}{c}\text { Somewhat } \\ \text { difficult }\end{array} & \begin{array}{c}\text { Very } \\ \text { difficult }\end{array} & \begin{array}{c}\text { Totally } \\ \text { unable }\end{array} \\ \square & \square & \square & \square & \square \\ \square & \square & \square & \square & \square \\ \square & \square & \square & \square & \square \\ \square & \square & \square & \square & \square \\ \square & \square & \square & \square & \square\end{array}$

\section{B Answer questions in this box if you have a few problems walking.}

During the past 4 weeks, how difficult would it have been for you to...

1. Walk 100 yards (about 1 city block)

2. Walk briskly

3. Walk 1 mile (about 20 minutes)

4. Be on your feet for 30 minutes

5. Climb 2 flights of stairs (about 20 stairs)
Not difficult at all

A little difficult

$\begin{array}{ll}\square & \square \\ \square & \square \\ \square & \square \\ \square & \square \\ \square & \square\end{array}$

Somewhat difficult

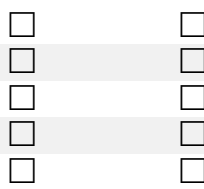

Very difficult

Totally unable

$\begin{array}{lll}\square & \square & \square \\ \square & \square & \square \\ \square & \square & \square \\ \square & \square & \square \\ \square & \square & \square\end{array}$

\section{Answer questions in this box if you have a lot of problems walking.}

During the past 4 weeks, how difficult was it for you to...

1. Walk 20 yards outside your home

2. Walk 100 yards (about 1 city block)

3. Walk between rooms

4. Be on your feet for 30 minutes

5. Climb 5 steps

\section{Not difficult \\ A little \\ Somewhat} at all

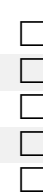
difficult

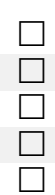
difficult

Very Totally difficult unable

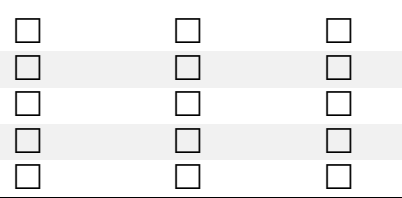

\section{Answer questions in this box if you are unable to walk.}

During the past 4 weeks, how difficult was it for you to...

Not difficult at all

1. Walk a few steps

2. Climb 2 steps

3. Stand without any help or support

4. Stand with the help of another person

5. Sit up in your bed

at all difficult

Somewhat difficult

Very difficult

Totally

$\begin{array}{lllll}\square & \square & \square & \square & \square \\ \square & \square & \square & \square & \square \\ \square & \square & \square & \square & \square \\ \square & \square & \square & \square & \square \\ \square & \square & \square & \square & \square\end{array}$




Attention: Check GO TO instructions for this question. Please read the statements
below and check the one that describes you best in the past 4 weeks.
$\begin{array}{lr}\text { I have no problems handling objects } & \rightarrow \text { Go to Box A on this page } \\ \text { I have a few problems handling objects } & \rightarrow \text { Go to Box B on this page } \\ \text { I have a lot of problems handling objects } & \square \rightarrow \text { Go to Box C on this page } \\ \text { I am unable to handle objects } & \rightarrow \quad \rightarrow \text { Go to Box D on this page }\end{array}$

\section{Please answer only questions in one of the boxes below, then move to next page}

\begin{tabular}{|lccccc|}
\hline \multicolumn{1}{|c}{ A Answer questions in this box if you have no problems handling objects. } \\
\hline $\begin{array}{l}\text { During the past } 4 \text { weeks, how difficult would it have been } \\
\text { for you to... }\end{array}$ & $\begin{array}{c}\text { Not difficult } \\
\text { at all }\end{array}$ & $\begin{array}{c}\text { A little } \\
\text { difficult }\end{array}$ & $\begin{array}{c}\text { Somewhat } \\
\text { difficult }\end{array} \quad \begin{array}{c}\text { Very } \\
\text { difficult }\end{array}$ & $\begin{array}{c}\text { Totally } \\
\text { unable }\end{array}$ \\
1. Place a 10-pound object on a shelf above your head & $\square$ & $\square$ & $\square$ & $\square$ & $\square$ \\
2. Push or pull open a heavy door & $\square$ & $\square$ & $\square$ & $\square$ & $\square$ \\
3. Lift and move heavy furniture (e.g., a sofa) & $\square$ & $\square$ & $\square$ & $\square$ & $\square$ \\
4. Open a new jar of food & $\square$ & $\square$ & $\square$ & $\square$ \\
5. Carry an object weighing 100 lbs. for 20 yards & $\square$ & $\square$ & $\square$ & $\square$ & $\square$ \\
\hline
\end{tabular}

\begin{tabular}{lcccc}
\hline \multicolumn{1}{c}{ B } & Answer questions in this box if you have a few problems \\
\hline
\end{tabular}

\begin{tabular}{|lccccc|}
\hline \multicolumn{1}{|c}{ C Answer questions in this box if you have a lot of problems } & handling objects. \\
\hline During the past 4 weeks, how difficult was it for you to: & Not difficult & A little & Somewhat & Very & Totally \\
unable & at all & difficult & difficult & difficult & $\square$ \\
1. Turn a key in a lock & $\square$ & $\square$ & $\square$ & $\square$ & $\square$ \\
2. Open a new milk carton & $\square$ & $\square$ & $\square$ & $\square$ & $\square$ \\
3. Open car doors & $\square$ & $\square$ & $\square$ & $\square$ \\
4. Push a button (e.g., on a telephone or TV remote control) & $\square$ & $\square$ & $\square$ & $\square$ & $\square$ \\
5. Eat with a knife and fork & $\square$ & $\square$ & $\square$ & $\square$ & $\square$ \\
\hline
\end{tabular}

\begin{tabular}{|lccccc|}
\hline \multicolumn{1}{|c}{ D Answer questions in this box if you are unable to handle objects. } & & \\
\hline During the past 4 weeks, how difficult was it for you to... & Not difficult & A little & Somewhat & Very & Totally \\
unable \\
difficult & difficult & difficult & $\square$ & $\square$ \\
1. Open car doors & $\square$ & $\square$ & $\square$ & $\square$ & $\square$ \\
2. Push a button (e.g., on a telephone or TV remote control) & $\square$ & $\square$ & $\square$ & $\square$ & $\square$ \\
3. Fasten your clothes (e.g., buttons or zippers) & $\square$ & $\square$ & $\square$ & $\square$ \\
4. Shake hands with people & $\square$ & $\square$ & $\square$ & $\square$ & $\square$ \\
5. Straighten or bend your arms & $\square$ & $\square$ & $\square$ & $\square$ & $\square$ \\
\hline
\end{tabular}




\section{Attention: Check GO TO instructions for this question. Please read the statements below and check the one that describes you best in the past 4 weeks.}

I have no problems doing usual daily activities

I have a few problems doing usual daily activities

I have a lot of problems doing usual daily activities

I am unable to doing usual daily activities $\rightarrow$ Go to Box A on this page

$\rightarrow$ Go to Box B on this page

$\rightarrow$ Go to Box C on this page

$\rightarrow$ Go to Box $D$ on this page

Please answer only questions in one of the boxes below, then move to next page A Answer questions in this box if you have no problems doing usual daily activities.

During the past 4 weeks, how difficult would it have been for you to...

Not difficult at all

1. Participate in physical leisure activities (e.g., exercise, gardening)

2. Accomplish more than usual in your work, school or other activities

3. Perform heavy household chores (e.g., scrubbing floors)

4. Go shopping for groceries

5. Accomplish as much as usual in your work, school or other activities

$\begin{array}{lllll}\square & \square & \square & \square & \square \\ \square & \square & \square & \square & \square \\ \square & \square & \square & \square & \square \\ \square & \square & \square & \square & \square \\ \square & \square & \square & \square & \square\end{array}$

\section{B Answer questions in this box if you have a few problems doing usual daily activities.}

During the past 4 weeks, how difficult would it have been for you to...

1. Go shopping for groceries

2. Perform your normal work or other daily activities

3. Participate in physical leisure activities (e.g., exercise, gardening)

4. Participate fully in your social or family life

5. Perform heavy household chores (e.g., scrubbing floors)

$\begin{array}{ccccc}\begin{array}{c}\text { Not difficult } \\ \text { at all }\end{array} & \begin{array}{c}\text { A little } \\ \text { difficult }\end{array} & \begin{array}{c}\text { Somewhat } \\ \text { difficult }\end{array} & \begin{array}{c}\text { Very } \\ \text { difficult }\end{array} & \begin{array}{c}\text { Totally } \\ \text { unable }\end{array} \\ \square & \square & \square & \square & \square \\ \square & \square & \square & \square & \square \\ \square & \square & \square & \square & \square \\ \square & \square & \square & \square & \square \\ \square & \square & \square & \square & \square\end{array}$

\begin{tabular}{|c|c|c|c|c|c|}
\hline \multicolumn{6}{|c|}{$\begin{array}{l}\text { Answer questions in this box if you have a lot of problems doing usual daily } \\
\text { activities. }\end{array}$} \\
\hline During the past 4 weeks, how difficult was it for you to... & $\begin{array}{l}\text { Not difficult } \\
\text { at all }\end{array}$ & $\begin{array}{l}\text { A little } \\
\text { difficult }\end{array}$ & $\begin{array}{c}\text { Somewhat } \\
\text { difficult }\end{array}$ & $\begin{array}{l}\text { Very } \\
\text { difficult }\end{array}$ & $\begin{array}{l}\text { Totally } \\
\text { unable }\end{array}$ \\
\hline 1. Go shopping for groceries & $\square$ & $\square$ & $\square$ & $\square$ & $\square$ \\
\hline 2. Perform light household chores (e.g., doing dishes) & $\square$ & $\square$ & $\square$ & $\square$ & $\square$ \\
\hline 3. Prepare your own meals & $\square$ & $\square$ & $\square$ & $\square$ & $\square$ \\
\hline
\end{tabular}

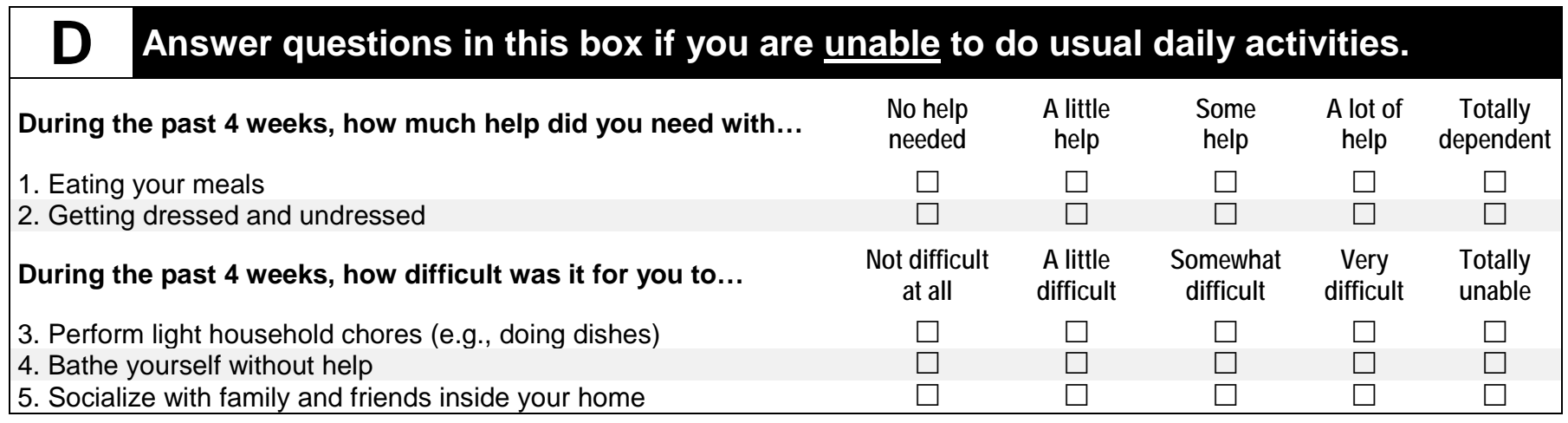




\section{Attention: Check GO TO instructions for this question. Please read the statements below and check the one that describes you best in the past 4 weeks.}

I have no pain or discomfort

I have mild pain or discomfort

I have moderate pain or discomfort

I have severe pain or discomfort $\rightarrow$ Go to Box $A$ on this page

$\rightarrow$ Go to Box $B$ on this page

$\rightarrow$ Go to Box C on this page

$\rightarrow$ Go to Box D on this page

\section{Please answer only questions in one of the boxes below, then move to next page}

\section{A Answer questions in this box if you have no pain or discomfort.}

During the past 4 weeks, how much of the time did you...

1. Feel great physically

2. Avoid strenuous activities for fear of pain

During the past 4 weeks, how much did pain (or discomfort)...

$\begin{array}{lll}\begin{array}{l}\text { None of } \\ \text { the little of }\end{array} & \begin{array}{l}\text { Some of } \\ \text { the time }\end{array}\end{array}$

3. Interfere with your physical leisure activities (e.g., exercise, gardening)

4. Limit you in doing things you wanted to do

5. Affect your ability to participate with enjoyment in strenuous leisure activities (e.g., sports)

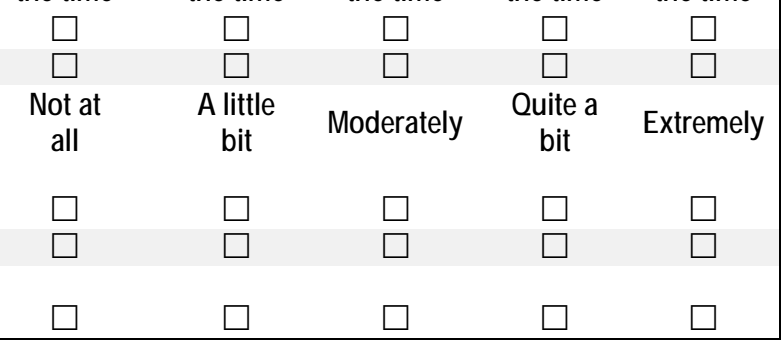

\section{B Answer questions in this box if you have mild pain or discomfort.}

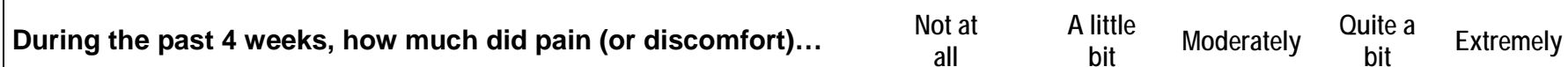

1. Interfere with your normal work or other daily activities

2. Interfere with your physical leisure activities (e.g., exercise, gardening)

3. Limit you in doing things you wanted to do

4. Interfere with your heavy household chores (e.g., scrubbing floors)

5. Interfere with your social activities with family or friends outside your home

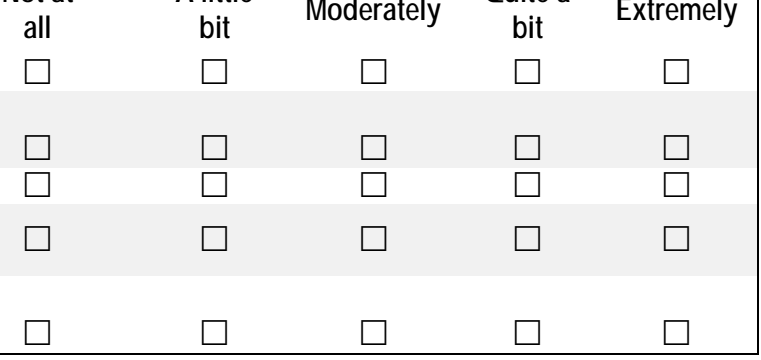

\begin{tabular}{|c|c|c|c|c|c|}
\hline During the past 4 weeks, how much did pain (or discomfort)... & $\begin{array}{l}\text { Not at } \\
\text { all }\end{array}$ & $\begin{array}{c}\text { A little } \\
\text { bit }\end{array}$ & Moderately & $\begin{array}{l}\text { Quite a } \\
\text { bit }\end{array}$ & Extremely \\
\hline 1. Interfere with your normal work or other daily activities & $\square$ & $\square$ & $\square$ & $\square$ & $\square$ \\
\hline 2. Restrict your routine daily activities & $\square$ & $\square$ & $\square$ & $\square$ & $\square$ \\
\hline $\begin{array}{l}\text { 3. Interfere with your physical leisure activities (e.g., exercise, } \\
\text { gardening) }\end{array}$ & $\square$ & $\square$ & $\square$ & $\square$ & $\square$ \\
\hline $\begin{array}{l}\text { 4. Interfere with your social activities with family or friends inside your } \\
\text { home }\end{array}$ & $\square$ & $\square$ & $\square$ & $\square$ & $\square$ \\
\hline 5. Frustrate you & $\square$ & $\square$ & $\square$ & $\square$ & $\square$ \\
\hline
\end{tabular}

\section{Answer questions in this box if you have severe pain or discomfort.}

During the past 4 weeks, how much did pain (or discomfort)...

Not at

1. Interfere with your social activities with family or friends outside your home

2. Restrict your routine daily activities

$\begin{array}{ccccc}\text { Not at } & \text { A little } & \text { Moderately } & \begin{array}{c}\text { Quite a } \\ \text { bit }\end{array} & \text { bit }\end{array}$ Extremely

$\begin{array}{ccccc}\square & \square & \square & \square & \square \\ \square & \square & \square & \square & \square\end{array}$




\section{Attention: Check GO TO instructions for this question. Please read the statements} below and check the one that describes you best in the past 4 weeks.

I am very happy

I am quite happy

I am somewhat unhappy

I am very unhappy $\rightarrow$ Go to Box A on this page

$\rightarrow$ Go to Box B on this page

$\rightarrow$ Go to Box C on this page

$\rightarrow$ Go to Box D on this page

\section{Please answer only questions in one of the boxes below, then move to next page}

\section{A Answer questions in this box if you are very happy.}

During the past 4 weeks, how much of the time did you...

1. Feel that thing were going your way

2. Feel cheerful

3. Feel sad

4. Feel positive about the future

5. Feel elated or overjoyed

$\begin{array}{ccccc}\begin{array}{c}\text { None of } \\ \text { the time }\end{array} & \begin{array}{c}\text { A little of } \\ \text { the time }\end{array} & \begin{array}{c}\text { Some of } \\ \text { the time }\end{array} & \begin{array}{c}\text { Most of } \\ \text { the time }\end{array} & \begin{array}{c}\text { All of } \\ \text { the time }\end{array} \\ \square & \square & \square & \square & \square \\ \square & \square & \square & \square & \square \\ \square & \square & \square & \square & \square \\ \square & \square & \square & \square & \square \\ \square & \square & \square & \square & \square\end{array}$

\section{B Answer questions in this box if you are quite happy.}

During the past 4 weeks, how much of the time did you...

\section{None of} the time

1. Feel depressed

2. Look on the bright side of things

3. Feel very upset

4. Feel completely satisfied with your life

5. Take a positive attitude toward yourself

$\begin{array}{lllll}\square & \square & \square & \square & \square \\ \square & \square & \square & \square & \square \\ \square & \square & \square & \square & \square \\ \square & \square & \square & \square & \square \\ \square & \square & \square & \square & \square\end{array}$

\section{Answer questions in this box if you are somewhat unhappy.}

During the past 4 weeks, how much of the time did you...

1. Feel depressed

2. Look on the bright side of things

3. Feel that life is entirely hopeless

4. Feel very upset

5. Feel enthusiastic

$\begin{array}{ccccc}\begin{array}{c}\text { None of } \\ \text { the time }\end{array} & \begin{array}{c}\text { A little of } \\ \text { the time }\end{array} & \begin{array}{c}\text { Some of } \\ \text { the time }\end{array} & \begin{array}{c}\text { Most of } \\ \text { the time }\end{array} & \begin{array}{c}\text { All of } \\ \text { the time }\end{array} \\ \square & \square & \square & \square & \square \\ \square & \square & \square & \square & \square \\ \square & \square & \square & \square & \square \\ \square & \square & \square & \square & \square \\ \square & \square & \square & \square & \square\end{array}$

\section{Answer questions in this box if you are very unhappy.}

During the past 4 weeks, how much of the time did you...

1. Feel that life is entirely hopeless

2. Feel irritable

3. Feel scared or panicky for no good reason

4. Feel very frustrated

5. Find yourself wishing you were dead and away from it all
None of A little of the time the time

Some of

Most of the time

the time

All of

$\begin{array}{lllll}\square & \square & \square & \square & \square \\ \square & \square & \square & \square & \square \\ \square & \square & \square & \square & \square \\ \square & \square & \square & \square & \square \\ \square & \square & \square & \square & \square\end{array}$

